\title{
Effectiveness assessment of countermeasures against bioterrorist smallpox attacks in Japan using an individual-based model
}

\author{
Tomohiro Zenihana $\cdot$ Hirofumi Ishikawa
}

Received: 9 December 2008 / Accepted: 4 September 2009/Published online: 26 September 2009

(C) The Japanese Society for Hygiene 2009

\begin{abstract}
Objectives This study is aimed at the evaluation of countermeasures in terms of the total number of patients and deaths, the number of vaccine doses used, and the time for eradication as examined through simulations of the outbreak of smallpox following a bioterrorist attack in the center of Okayama City. Three countermeasures were considered: traced vaccination (TV), mass vaccination (MV), and school closure.

Methods An individual-based model was adopted, in which every individual is assigned their own personal information, behavioral pattern, and interactions among social settings to simulate the situation on a realistic basis. The influence of residual immunity obtained by past vaccination reflected age-dependent immunity to smallpox in Japan.

Results MV performed within a 2-day period at the same time in 10 school districts had the highest effectiveness in reducing the total number of patients and deaths among all simulations. Performing both TV and MV simultaneously was much more effective than performing TV or MV individually. The decrease in the number of patients with TV or MV in combination with school closure was faster than that by TV or MV without school closure.

Conclusions According to the simulations results, it was advisable to carry out MV, or both TV and MV, simultaneously with school closure as countermeasures against a smallpox epidemic initiated by a bioterrorist attack.
\end{abstract}

T. Zenihana $\cdot$ H. Ishikawa $(\bowtie)$

Department of Human Ecology,

Graduate School of Environment Science,

Okayama University, Okayama 700-8530, Japan

e-mail: ishikawa@ems.okayama-u.ac.jp
Keywords Smallpox · Bioterrorism ·

Individual-based model (IBM) · Residual immunity · Countermeasures

\section{Introduction}

In recent years, numerous terrorist attacks have occurred around the world. The weapons used in bioterrorism are easily and inexpensively manufactured; thus bioterrorism is regarded as a significant threat. Smallpox is monitored as a dangerous virus because the US Centers for Disease Control and Prevention (CDC) have shown smallpox to be a biological weapon with high infectiousness and mortality [1]. The World Health Organization (WHO) declared that smallpox was eradicated from the whole world in 1980. Because smallpox was eradicated from Japan in 1956, regular vaccination against smallpox ended in Japan in 1976 [2]. Therefore, the population of nonimmune young people has increased since then [3].

The present study attempted to predict the effectiveness of countermeasures against bioterrorism and the scale of loss through simulations of various scenarios of bioterrorism using smallpox. The center of Okayama City, the capital of Okayama Prefecture in the western part of the main island of Japan, which has a population of about 400,000 , was chosen as the target region.

Fenner et al. [4] studied the symptoms of smallpox and the history of its eradication. There have been a number of studies on bioterrorism using smallpox on the basis of mathematical models. Kaplan et al. [5] examined the effectiveness of traced vaccination (TV) and mass vaccination (MV) strategies using a deterministic model. In TV, which is recommended by the CDC, patients are traced and persons who came into contact with those patients are 
vaccinated [6], while in MV, all persons are vaccinated in a certain region. Nishiura et al. [7] investigated the influence of residual immunity in Japan by means of a deterministic model. Kretzschmar et al. [8] took advantage of the basic reproductive number and the effective reproductive number by means of a stochastic model. Longini et al. [9] set the behavioral pattern for each level of disease severity, and Eubank et al. [10] set behavioral patterns dependent on age groups and social setting. Riley et al. [11] investigated countermeasures for the whole of Great Britain.

In this article, a smallpox outbreak in the center of Okayama City is examined using an individual-based model (IBM) in which every individual is assigned personal information such as age and home address, behavioral pattern such as movement between home and workplace, and interactions among social settings. In Japan, the majority of people born after 1977, between 1970 and 1976, and before 1969 have been vaccinated none, once, and more than two times, respectively [12]. The influence of residual immunity due to past vaccination was reflected in the severity of symptoms and the efficacy of vaccination.

The efficacies of various scenarios of a TV strategy with respect to the length of the necessary tracing period, of a MV strategy with respect to the number of school districts vaccinated at the same time and the time limit per school district, and of school closure against bioterrorism were examined. In addition, scenarios of carrying out both TV and MV simultaneously and in combination with school closure were investigated.

In this article, the efficacy of countermeasures in terms of the total number of patients and deaths, the number of vaccine doses used, and the time for eradication were verified by carrying out simulations of an outbreak of smallpox. According to the results of the simulations, although performing TV succeeded in gradually decreasing the number of patients, it was difficult to eliminate the smallpox epidemic rapidly, and the maximum success rate for elimination within 200 days in 300 trial simulations was assessed to be $63.3 \%$. The scenario of performing MV in 10 school districts at the same time within 2-day periods showed the highest effectiveness of all scenarios and the mean number of total patients and deaths, and the success rate for elimination within 200 days were 50.5, 8.3, and $100 \%$, respectively. The scenario of performing both TV and MV simultaneously was much more effective than the scenarios with TV or MV individually. In the scenario with school closure only, the epidemic reached a peak at 173 days, which was later than the no-intervention scenario. By performing TV or MV accompanied by school closure, the number of patients decreased more rapidly than with TV or MV individually. Consequently, performing $\mathrm{MV}$, or both TV and MV simultaneously, with school closure showed high effectiveness against a smallpox epidemic initiated by a bioterrorist attack.

\section{Materials and methods}

\section{Natural history of smallpox}

The progress of smallpox is separated into three stages, namely incubation, fever (prodromal), and rash periods [4]. Generally, the incubation, fever, and rash periods last 7-17, 1.5-4.5, and 4.5-12.5 days, with means of 12, 3, and 8.5 days, respectively [4]. Patients with smallpox in the early rash period have the highest infectiousness, while there is a low risk of infection just before the rash period [4]. Smallpox occasionally causes patient fatality at 717 days after fever or patients recover after the rash period [4].

In Japan, smallpox was eradicated in 1956 [2]. Consequently, the regular vaccination program for smallpox was ended in 1976. Therefore, the number of nonimmune young people has increased since then [3]. The majority of people born before 1969, between 1970 and 1976, and after 1977 have been vaccinated more than two, one, and zero times, respectively [12]. In 2004, Takeuchi et al. measured the antibody titer of smallpox in 120 individuals and reported that 67 of 83 individuals who had been vaccinated one or more times had 4 times neutralizing-antibody, especially 22 individuals had 32 times neutralizing-antibody, and that the other 37 individuals had not more 3.4 times neutralizing-antibody [13].

\section{Transmission model of smallpox}

In the model for the transmission of smallpox, the population was divided into five classes: negative, incubation, fever, rash, and recover. The patients were classified into three severity types: ordinary, modified, and hemorrhagic, according to severity of symptoms [4]. Patients of the modified type have lower infectiousness than those of the ordinary type, but modified-type patients may spread more smallpox infection because of the difficulty in recognizing them. Patients of the hemorrhagic type have severe symptoms, higher infectiousness, and higher mortality compared with ordinary-type patients [4].

Although Arita pointed out that the residual immunity derived from past vaccinations was indistinct [12], the present model supposed the effect of residual immunity on the suppression of infection or symptoms. In this model, it was considered that the immunity obtained by vaccination before 1976 persists to the present day [13]. Hence, the negative class (residual immunity class) was subdivided into three subclasses $(\mathrm{A}-\mathrm{C})$ according to the administration 
Table 1 Probability of severity types of symptoms due to residual immunity

\begin{tabular}{lllll}
\hline $\begin{array}{l}\text { Negative } \\
\text { subclass }\end{array}$ & $\begin{array}{l}\text { Full } \\
\text { protection }(\%)\end{array}$ & \multicolumn{3}{l}{ Infection $(\%)$} \\
\cline { 3 - 5 } & & Modified & Ordinary & Hemorrhagic \\
\hline A & 10 & 80 & 10 & 0 \\
B & 0 & 30 & 67 & 3 \\
C & 0 & 0 & 95 & 5 \\
\hline
\end{tabular}

of previous vaccinations: (A) more than two vaccinations (born before 1969), (B) one vaccination (born between 1970 and 1976), and (C) no vaccinations (born after 1977). Each negative subclass had a different probability of infection on contact with an infectious patient [12]. Because of the high residual immunity of the negative subclass A, it was assumed that $10 \%$ of this subclass have full protection, and that $80 \%, 10 \%$, and $0 \%$ developed modified-, ordinary-, and hemorrhagic-type smallpox, respectively, while because of low residual immunity in negative subclass B, it was assumed that $30 \%, 67 \%$, and $3 \%$ developed modified-, ordinary-, and hemorrhagic-type smallpox, respectively. For negative subclass $\mathrm{C}$, it was assumed that $95 \%$ and 5\% developed ordinary- and hemorrhagic-type smallpox, respectively (Table 1).

The length of the incubation period was assumed to be dependent on severity type $(\mathrm{A}-\mathrm{C})$, and was set at 10 14 days for ordinary and modified type and 8-15 days for hemorrhagic type (Table 2). The fever and rash periods were fixed at 3 and 9 days, respectively, under the assumption that the fever and rash periods are independent of severity type.

The infectiousness of smallpox is dependent on both severity type and progress of symptoms. Patients in the early rash period had the highest infectiousness, while those in the fever period had low infectiousness [4]. In the model, the infectious period lasted 2-12 days from the 2 nd day of the fever stage. Two factors related to infectiousness were introduced: "base" and "multiplier" [14]. The baseline of infectiousness was set as the infectiousness of the ordinary type on the 2nd day of the fever stage. "Multiplier" stands for the relative factor of the infectiousness of modified and hemorrhagic types compared with the baseline (infectiousness of the ordinary type), which were set at 0.33 and 5, respectively (Table 3 ).

Death from smallpox occurred after a 7-12 days incubation period. Mortality from smallpox was set as 0.1, 0.3, and 1 according to the severity types of modified, ordinary, and hemorrhagic, respectively $[4,9]$.

The model scheme for the transmission dynamics of smallpox is shown in Fig. 1.
Table 2 Distributions of incubation period by severity type

\begin{tabular}{lcc}
\hline $\begin{array}{l}\text { Incubation } \\
\text { period (days) }\end{array}$ & $\begin{array}{l}\text { Modified and } \\
\text { ordinary }(\%)\end{array}$ & $\begin{array}{l}\text { Hemorrhagic } \\
(\%)\end{array}$ \\
\hline 8 & - & 3.6 \\
9 & - & 10.9 \\
10 & 16.7 & 16.5 \\
11 & 27.8 & 19.4 \\
12 & 33.3 & 20.2 \\
13 & 16.7 & 17.7 \\
14 & 5.5 & 7.3 \\
15 & - & 4.4 \\
\hline
\end{tabular}

Table 3 Relative infectiousness of smallpox

\begin{tabular}{lll}
\hline Severity type & Base & Multiplier \\
\hline Ordinary & 0.07 & 1.00 \\
Modified & - & 0.33 \\
Hemorrhagic & - & 5.00 \\
\hline Days after incubation period & Infectiousness \\
\hline$\leq 1$ & 0.0 \\
2 & $1.0 \times$ base $\times$ multiplier \\
3 & $2.0 \times$ base $\times$ multiplier \\
$4-8$ & $4.0 \times$ base $\times$ multiplier \\
9 & $3.0 \times$ base $\times$ multiplier \\
10 & $2.0 \times$ base $\times$ multiplier \\
$11-12$ & $1.0 \times$ base $\times$ multiplier \\
$13 \leq$ & 0.0 \\
\hline
\end{tabular}

Structure of the targeted area

In this article, the center of Okayama City, which is the capital of Okayama Prefecture in the western part of the main island of Japan and had a population of 412,992 in 2006 [15], was chosen as a target region to simulate an outbreak of smallpox. The center of Okayama City was divided into its 37 school districts, and all the school districts were assigned a population on the basis of the demographic census [15]. Crowds of people from all the school districts gather in the central school district where the central station and many shopping malls are located.

The age distribution in Okayama City in 2006 is shown in Fig. 2; the sex ratio of males to females in Okayama City was 48:52 [15]. It follows from the age distribution in Okayama City that the proportions of three subclasses (A-C) were estimated as $32.9 \%, 11.2 \%$, and $55.9 \%$, respectively [15]. 


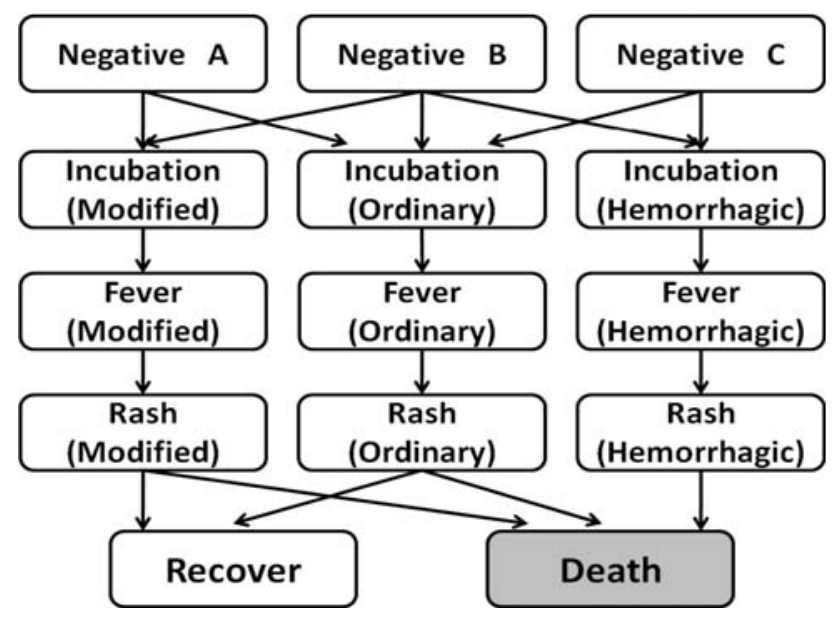

Fig. 1 Model scheme for transmission dynamics of smallpox
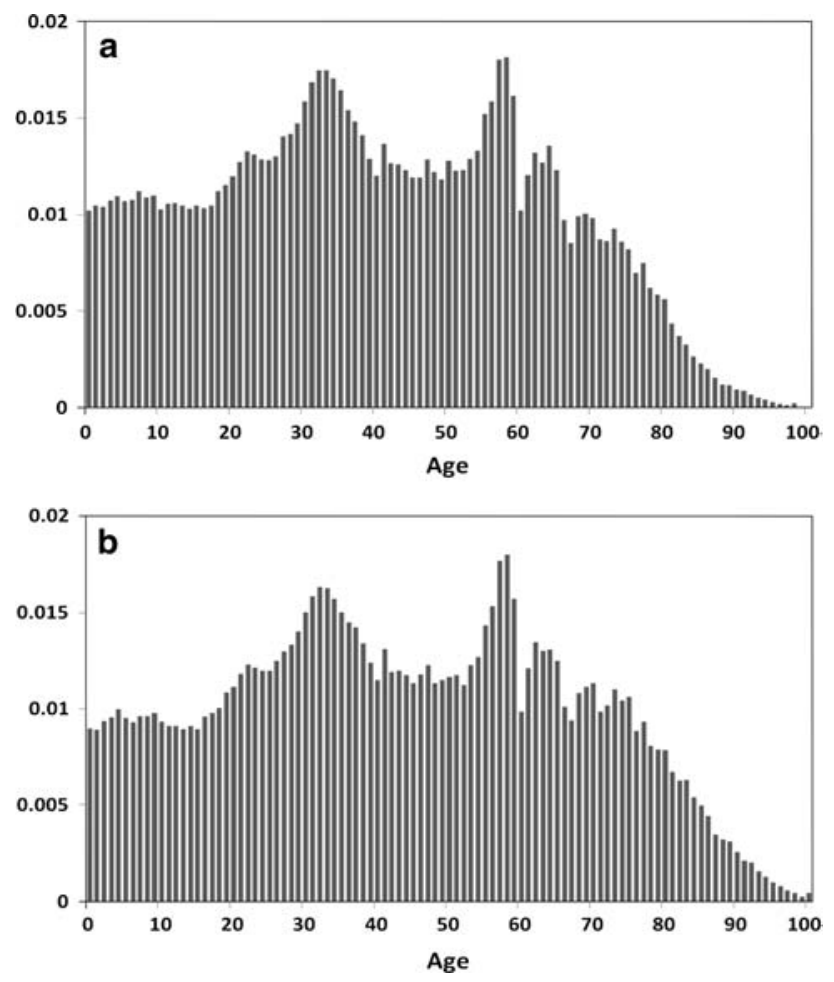

Fig. 2 Age distribution of Okayama City in 2006 [15] a male, b female

There were 169,270 households in the targeted region [15]. In this article, it was assumed that each household was composed of 1-7 individuals, with the proportions as set out in Table 4, i.e., one-person households were composed of one-person more than 18 years old, two-person households were composed of a couple of a man and a woman more than 18 years old and within 5 years disparity in age, and those households with more than three persons were composed of a couple and their children or parents
Table 4 Ratios of households by size

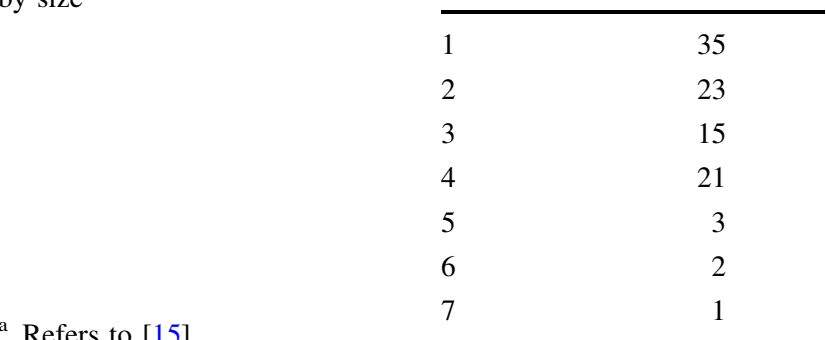

${ }^{a}$ Refers to [15]

Table 5 Ratios and ages of roles

\begin{tabular}{lll}
\hline Role & Age (years) & Ratio $^{\mathrm{a}}(\%)$ \\
\hline Nonworker & $\geq 4,16 \leq$ & 20 \\
Worker & $16 \leq$ & 68 \\
Student & $5 \leq$ & 10 \\
HCW & $22 \leq$ & 2 \\
\hline
\end{tabular}

a Refers to [15]

with more than 20 years disparity in age between generations.

Individuals were classified into four roles: nonworker, worker (except for healthcare worker), student, and healthcare worker $(\mathrm{HCW})$, the proportions of which were estimated as $20 \%, 68 \%, 10 \%$, and $2 \%$, respectively (Table 5) [15].

In this article, three kinds of social settings: school (kindergarten, elementary, junior high, high, and college), workplace, and hospital (only general hospital) were considered. The numbers of these social settings were estimated from the demographic census [15]. The population size of workplaces was assumed to be 15 persons to generate an appropriate mass of close contact. Students of ages $5-6,7-12,13-15,16-18$, and 19 years or over attend kindergarten, elementary, junior high, high, and college, respectively (Table 6).

Behavioral patterns of the population in the targeted region

In this article, it was supposed that "close contact" occurs in a social setting and that "casual contact" occurs accidentally during trips or shopping. The relative infectiousness in each contact is tabulated as compared with that in household contact in Table 7. It was assumed that students in primary school-college belonged to classes of 30 persons, and that the risk of infection in close contact in school decreased by degrees within the class, the age group, and the overall size of the school, while only one class was set per kindergarten. A very low probability was set for the risk of infection from casual contacts [4]. The relative risk of infection from casual contacts between residents of a 
Table 6 Number of social settings in the center of Okayama City

\begin{tabular}{lrl}
\hline Social setting & Number & Age range (years) \\
\hline School $^{\mathrm{a}}$ & & \\
Kindergarten $_{\text {Elementary }}$ & 44 & $5-6$ \\
Junior high & 37 & $7-12$ \\
High & 18 & $13-15$ \\
College & 16 & $16-18$ \\
Workplace $_{\text {Hospital }}^{\mathrm{a}}$ & 6 & $19 \leq$ \\
\end{tabular}

${ }^{\mathrm{a}}$ Refers to [15]

Table 7 Relative risk of infection for contacts as compared with household contacts

\begin{tabular}{ll}
\hline Contact & $\begin{array}{l}\text { Relative risk } \\
\text { of infection }\end{array}$ \\
\hline Close & \\
Household & 1.0 \\
Kindergarten & \\
$\quad$ Same grade & 0.7 \\
Different grade & 0.07 \\
Elementary & \\
Same class & 0.7 \\
Same grade & 0.07 \\
Different grade & 0.035 \\
Junior high school & \\
Same class & 0.7 \\
Same grade & 0.07 \\
Different grade & 0.035 \\
High school & \\
Same class & 0.7 \\
Same grade & 0.07 \\
Different grade & 0.035 \\
College & \\
Same class & 0.5 \\
Different group & 0.004 \\
Workplace & 0.7 \\
Casual & \\
Central school district & 0.0001 \\
Other school district & \\
\hline & \\
&
\end{tabular}

school district and a patient was considered for three cases: (a) staying within their own school district, (b) visiting other school districts except for the central school district, and (c) visiting the central school district. In-hospital infection was not considered.

Longini et al. [9] and Burke et al. [14] considered a change in the behavioral pattern of patients according to
Table 8 Behavioral patterns of patients in fever and rash periods

\begin{tabular}{llcc}
\hline Type & $\begin{array}{l}\text { Second and third } \\
\text { day of fever }(\%)\end{array}$ & $\begin{array}{l}\text { At the end of } \\
\text { first day of rash }\end{array}$ \\
\hline Ordinary & Go to hospital & 47.5 & $\begin{array}{c}\text { All patients go } \\
\text { to hospital }\end{array}$ \\
& Withdraw to home & 47.5 & \\
Modified & Normal behavior & 5 & \\
& Go to hospital & 25 & \\
& Withdraw to home & 25 & \\
& Normal behavior & 50 & \\
\hline
\end{tabular}

their disease severity. So, a different behavioral pattern was set for patients than for nonpatients (Table 8) [9, 14].

It was assumed that $20 \%$ of $\mathrm{HCW}$ were engaged in the administration of vaccination for $8 \mathrm{~h}$ a day, and that the number of administrations of vaccine per HCW was 6 per hour. Hence, the maximum number of administrations of vaccine per hour was estimated as follows:

(Maximum number of administrations of vaccine per hour) $=($ Number of $\mathrm{HCW}) \times 0.2 \times 6 \times 8 / 24$.

An IBM targeting the population of 412,992 was constructed by setting the above behavioral patterns.

\section{Vaccination priority}

It is natural to determine a vaccination priority with consideration of residual immunity [12]. On index patients being recognized, the present model adopted the following vaccination priority: (1) HCW, (2) residual immunity subclass C, (3) residual immunity subclass B, and (4) residual immunity subclass $\mathrm{A}$.

Efficacy of vaccination

The LC16m8 strain that is used for smallpox vaccine in Japan has a protective efficacy of almost $100 \%$ in nonpatients, high efficacy in patients within 4 days after infection, and low efficacy at 4-7 days after infection, but almost no efficacy from 8 days after infection [3]. It was assumed that the efficacy of vaccination depended on the time of vaccination and residual immunity subclass, i.e., the efficacy of vaccination reduced in the order $\mathrm{A}, \mathrm{B}$, and $\mathrm{C}$ (Table 9).

A mortality of $10^{-6}$ among known side-effects of the LC16m8 strain was considered [3].

\section{Countermeasures}

This article covers a TV strategy, MV strategy, and school closure as countermeasures. It was assumed that these countermeasures would be carried out for 5 days from the 
Table 9 Efficacy of vaccination

\begin{tabular}{|c|c|c|c|}
\hline Time of vaccination & $\begin{array}{l}\text { Residual } \\
\text { immunity } \\
\text { subclass }\end{array}$ & Symptom & $\begin{array}{l}\text { Efficacy } \\
(\%)\end{array}$ \\
\hline \multirow[t]{5}{*}{ Before infection } & A & Full protection & 100 \\
\hline & $\mathrm{B}$ & Full protection & 99 \\
\hline & & Modified & 1 \\
\hline & $\mathrm{C}$ & Full protection & 97 \\
\hline & & Modified & 3 \\
\hline \multirow[t]{5}{*}{4 days after infection } & A & Full protection & 100 \\
\hline & $\mathrm{B}$ & Full protection & 95 \\
\hline & & Modified & 5 \\
\hline & $\mathrm{C}$ & Full protection & 90 \\
\hline & & Modified & 10 \\
\hline \multirow[t]{8}{*}{ 5-7 days after infection } & A & Full protection & 20 \\
\hline & & Modified & 80 \\
\hline & $\mathrm{B}$ & Full protection & 10 \\
\hline & & Ordinary & 20 \\
\hline & & Modified & 70 \\
\hline & $\mathrm{C}$ & Ordinary & 38 \\
\hline & & Modified & 60 \\
\hline & & Hemorrhagic & 2 \\
\hline From 8 day after infection & $\mathrm{A}-\mathrm{C}$ & & 0 \\
\hline
\end{tabular}

identification of the index patient. In the TV strategy, persons who have probably come into contact with patients diagnosed with smallpox were traced and vaccinated. Possible tracing to the household and the social setting of the patients was limited to success rates of 0.9 and 0.7 , respectively, while it was impossible for casual contacts to be traced. It was studied whether an increase in the necessary tracing period from 1 to 3 days would influence the smallpox epidemic.

On the other hand, a MV strategy for the people in the area of the outbreak of smallpox was also assessed. The MV program would be executed for every school district. It was examined how the shift in the number of school districts performing MV at the same time from 1 to 2 or more, or a shift in the time period of MV from 1 to 2 days, would influence a smallpox epidemic from the viewpoint of HCW resources. After the time limit of the MV program in one school district, the MV program would move to another school district.

Moreover, the influence of school closure on the epidemic was investigated, which was continued until the eradication of smallpox.

\section{Results}

To examine the influence of TV and MV strategies and school closure on smallpox epidemics, 300 trial stochastic
Table 10 Scenarios

\begin{tabular}{lllll}
\hline Scenario & $\begin{array}{l}\text { Necessary } \\
\text { tracing } \\
\text { period in } \\
\text { TV (days) }\end{array}$ & $\begin{array}{l}\text { Number of school } \\
\text { districts performing } \\
\text { MV at the same } \\
\text { time }\end{array}$ & $\begin{array}{l}\text { Time limit of } \\
\text { MV per } \\
\text { school district } \\
\text { (days) }\end{array}$ & $\begin{array}{l}\text { School } \\
\text { closure }\end{array}$ \\
\hline$(1)$ & - & - & - & - \\
$(2)$ & 1 & - & - & - \\
$(3)$ & 2 & - & - & - \\
$(4)$ & 3 & - & - & - \\
$(5)$ & - & 1 & 1 & - \\
$(6)$ & - & 5 & 1 & - \\
$(7)$ & - & 10 & 1 & - \\
$(8)$ & - & 1 & 2 & - \\
$(9)$ & - & 5 & 2 & - \\
$(10)$ & - & 10 & 2 & - \\
$(11)$ & 3 & 10 & 1 & - \\
$(12)$ & - & - & - & 0 \\
$(13)$ & 2 & - & - & 0 \\
$(14)$ & - & 1 & 2 & \\
\hline
\end{tabular}

simulations of 14 scenarios were carried out for smallpox epidemics initiated by a bioterrorist attack. The period of simulation was set as up to 200 days to assess the swift eradication of the epidemic. Twenty index patients were introduced in the beginning for every scenario.

In the baseline scenario 1 , there were no interventions. In scenarios in which only TV was performed (2-4), the necessary trace period was set to 1,2 , and 3 days. In scenarios in which only MV was performed (5-10), the number of school districts performing $\mathrm{MV}$ at the same time was changed among 1, 5, and 10, and also the time limit of MV in one school district from 1 to 2 days. The more school districts performing MV at the same time, the lower the number of doses of vaccine administered per day per school district because of the dispersion of HCWs. In scenario 11, both TV and MV were carried out simultaneously. In scenarios 12 14 , the influence of combining school closure with other strategies was studied. These scenarios are summarized in Table 10. Table 11 shows the mean numbers of total patients, total deaths, and total doses of vaccine administered with $95 \%$ confidence intervals, and the success rate of elimination within 200 days for the 14 scenarios.

\section{Scenario 1}

In the baseline scenario, a mean of $71.4 \%$ and $15.0 \%$ of the entire population in 300 trial simulations would be infected and die, respectively. It was impossible to eradicate smallpox within 200 days in any of the 300 trial simulations (Fig. 3). 
Table 11 Summary of results for the 14 investigated scenarios

\begin{tabular}{lcccr}
\hline Scenario & $\begin{array}{l}\text { Mean number of total } \\
\text { patients }(95 \% \text { CI })\end{array}$ & $\begin{array}{l}\text { Mean number of total } \\
\text { deaths }(95 \% \text { CI })\end{array}$ & $\begin{array}{l}\text { Mean total vaccine } \\
\text { doses (95\% CI) }\end{array}$ & $\begin{array}{l}\text { Success rate of elimination } \\
\text { within 200 days }(\%)\end{array}$ \\
\hline$(1)$ & $294,977.4(294,616.6-295,338.2)$ & $63,643.6(63,518.7-63,768.5)$ & $300,448.2(300,012.1-300,884.3)$ & 0.0 \\
$(2)$ & $269.6(243.2-295.9)$ & $31.6(28.8-34.3)$ & $37,103.3(35,805.5-38,401.1)$ & 63.3 \\
$(3)$ & $317.4(285.1-349.7)$ & $38.1(34.5-41.6)$ & $39,172.2(37,799.0-40,545.3)$ & 59.0 \\
$(4)$ & $417.5(369.9-465.0)$ & $58.9(52.6-65.3)$ & $41,930.2(40,347.7-43,512.7)$ & 52.7 \\
$(5)$ & $83.5(79.2-87.7)$ & $15.5(14.8-16.2)$ & $411,047.1(410,230.7-411,863.5)$ & 100.0 \\
$(6)$ & $55.3(52.4-58.1)$ & $8.8(8.3-9.2)$ & $388,804.2(388,799.6-388,808.8)$ & 100.0 \\
$(7)$ & $285.3(125.7-444.9)$ & $32.3(16.0-48.6)$ & $261,692.9(261,557.2-261,828.7)$ & 59.3 \\
$(8)$ & $331.7(286.5-376.8)$ & $64.5(55.0-73.9)$ & $408,053.3(406,083.3-410,023.4)$ & 100.0 \\
$(9)$ & $55.5(54.3-56.7)$ & $9.4(9.1-9.8)$ & $412,984.9(412,984.6-412,985.2)$ & 100.0 \\
$(10)$ & $50.5(49.7-51.4)$ & $8.3(8.0-8.6)$ & $409,985.3(409,980.2-409,990.3)$ & 100.0 \\
$(11)$ & $58.9(57.3-60.6)$ & $8.9(8.5-9.2)$ & $287,146.5(286,966.2-287,326.7)$ & 100.0 \\
$(12)$ & $23,115.2(21,622.8-24,607.7)$ & $2,761.1(2,581.6-2,940.5)$ & $22,878.5(21,846.8-23,910.1)$ & 0.0 \\
$(13)$ & $192.8(176.4-209.2)$ & $25.1(23.2-27.1)$ & $34,362.5(33,343.0-35,382.0)$ & 72.0 \\
$(14)$ & $117.1(99.2-135.0)$ & $25.6(21.8-29.4)$ & $406,721.3(398,486.0-414,956.6)$ & 100.0
\end{tabular}

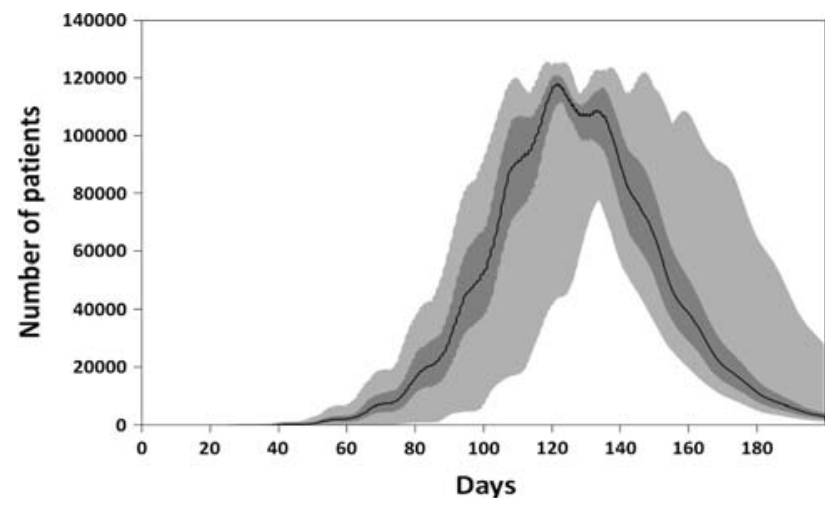

Fig. 3 Progression of the number of smallpox patients in scenario 1 . The black line shows the mean number of patients in 300 trials. The dark gray and light gray zones show the interquartile range and the whole range, respectively

\section{Scenarios 2-4}

The influence of a change in the necessary tracing period for TV was investigated (Fig. 4). The number of patients decreased gradually in every scenario. The total number of patients in the scenario with 3 days necessary for the tracing period for TV (scenario 4) would be more than those for tracing periods of 1 and 2 days (scenarios 2 and 3). Scenarios 2-4 succeeded in controlling the outbreak within 200 days at rates of $63.3 \%, 59.0 \%$, and $52.7 \%$ in 300 trial simulations, respectively.

\section{Scenarios 5-7}

The influence on the epidemic of changing the number of school districts performing MV at the same time was compared with a 1-day limit (Fig. 5). For 1 and 5 school districts performing MV at the same time (scenarios 5 and 6), the mean number of total patients was 83.5 and 55.3 and the mean number of total deaths was 15.5 and 8.8 , respectively, which showed the high effectiveness of the MV strategy in the reduction of the numbers of total patients and deaths. For 10 school districts performing MV at the same time (scenario 7), the number of patients was still increasing at 200 days after the outbreak. The success rate of elimination within 200 days for scenario 7 was assessed to be $59.3 \%$ in 300 trial simulations.

\section{Scenarios $8-10$}

The influence on the epidemic of altering the number of school districts performing MV at the same time with a 2day limit was compared (Fig. 6). All scenarios succeeded in eliminating the outbreak within 200 days in all 300 trial simulations. For 5 and 10 school districts performing MV at the same time (scenarios 9 and 10), the mean number of total patients was 55.5 and 50.5, and the mean number of total deaths was 9.4 and 8.3 , respectively. Scenario 10 showed the highest effectiveness in reducing the total number of patients and deaths among the 14 scenarios. For only one school district performing MV at the same time (scenario 8), the number of patients would increase at 82 days after the start of the outbreak and thereafter decrease rapidly.

\section{Scenario 11}

Scenarios performing TV or MV alone (scenarios 4 and 7) were compared with the scenario performing both TV and 

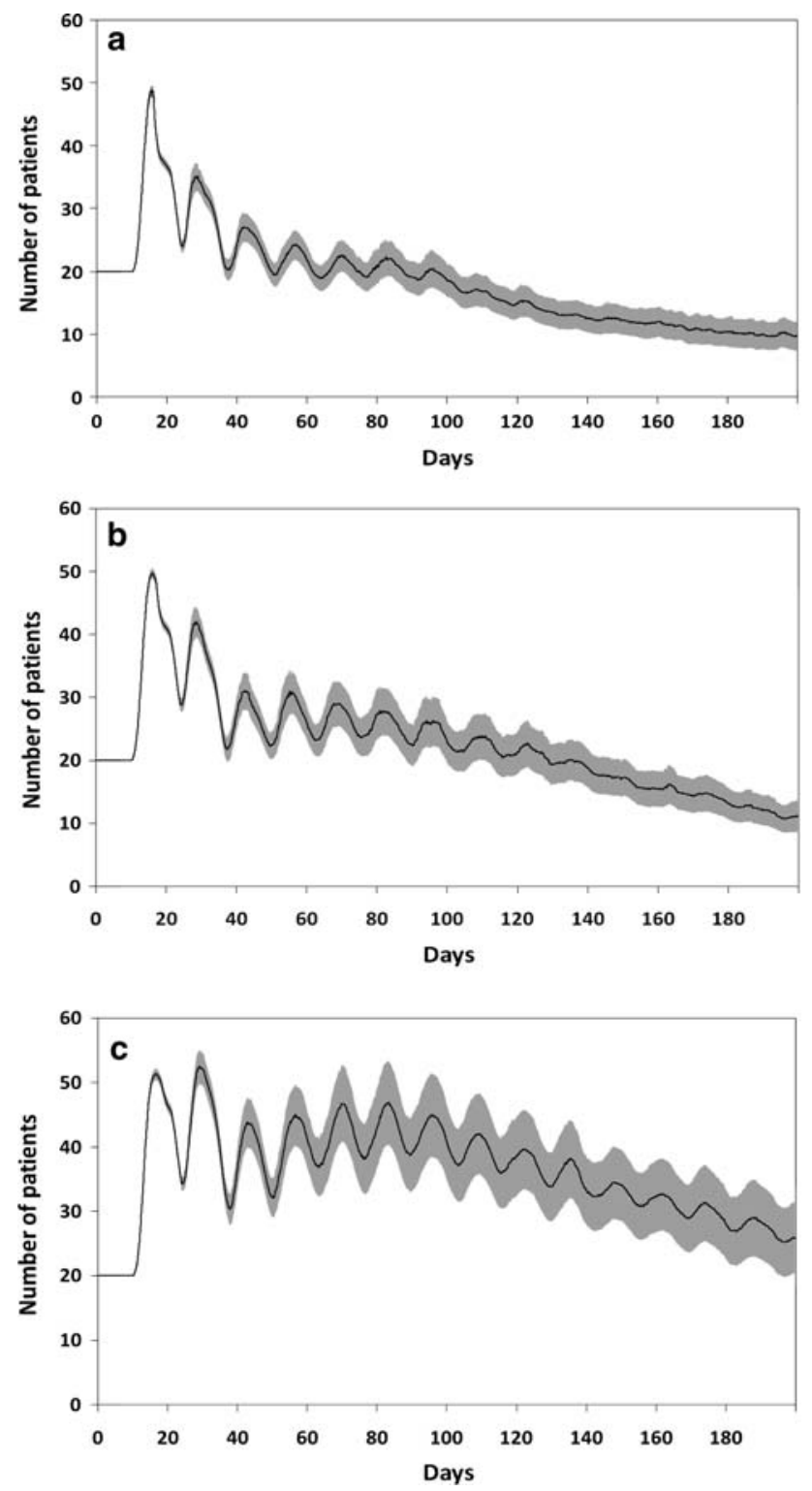

Fig. 4 Progression of the number of smallpox patients in scenarios 2-4. a-c The transmission in scenarios 2, 3, and 4, respectively. The black line shows the mean number of patients in 300 trials. The gray zone shows the $95 \%$ confidence interval

MV simultaneously (scenario 11) (Fig. 7). Scenario 11 was the combination of scenario 4 with scenario 7 . Scenarios 4 and 7 could not succeed in eliminating the outbreak within 200 days in 300 trial simulations. In scenario 11 , the mean numbers of total patients and deaths were 58.9 and 8.9 , respectively.

\section{Scenario 12}

Scenario 12 compared performing school closure only with the baseline scenario 1 (Fig. 8). In scenario 12, the number of patients was still increasing at 200 days after the start of

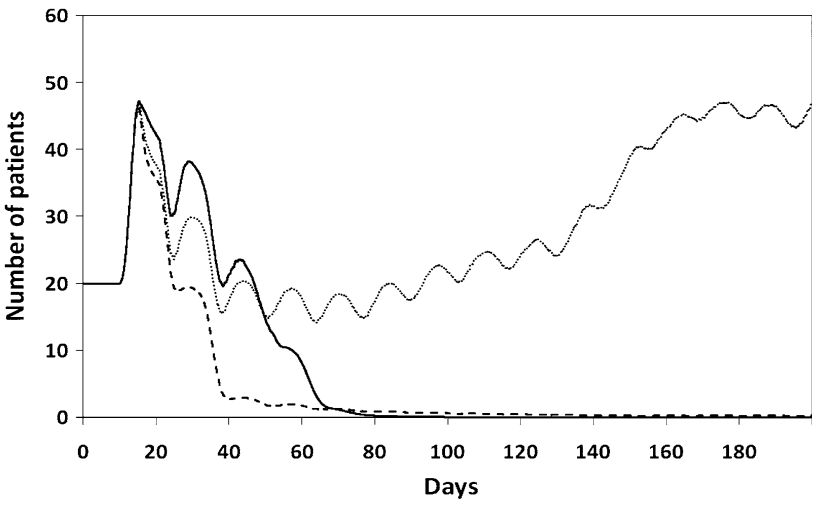

Fig. 5 Progression of the number of smallpox patients in scenarios 5-7. Solid, broken, and dashed lines show the mean number of patients in 300 trials in scenarios 5, 6, and 7, respectively

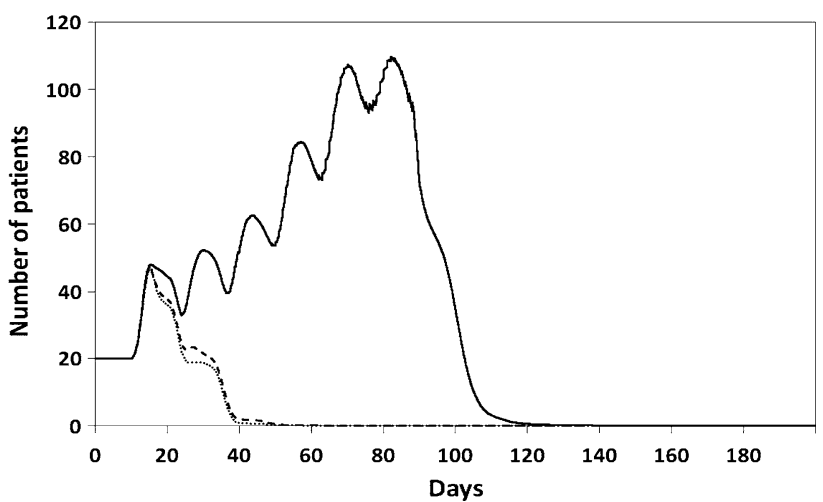

Fig. 6 Progression of the number of smallpox patients in scenarios 8-10. Solid, broken, and dashed lines show the mean number of patients in 300 trials in scenarios 8,9 , and 10 , respectively

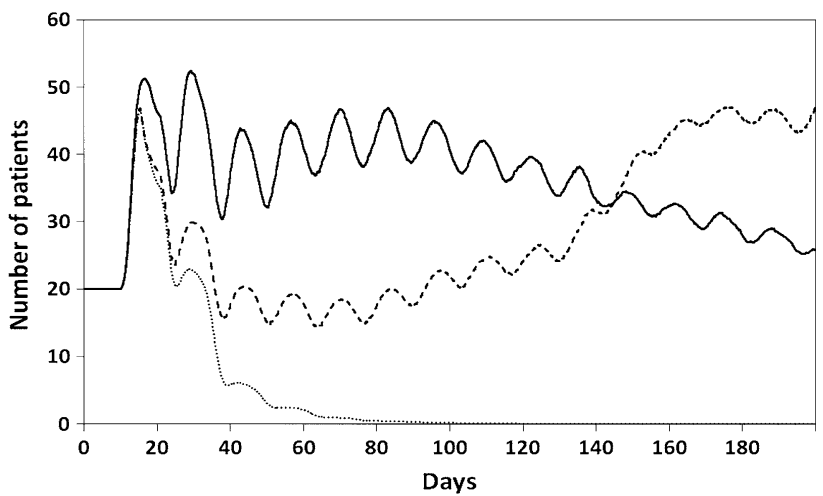

Fig. 7 Progression of the number of smallpox patients in scenarios 4, 7, and 11. Solid, broken, and dashed lines show the mean number of patients in 300 trials in scenarios 4,7 , and 11 , respectively

the outbreak, and it was impossible to eradicate the outbreak within 200 days in 300 trial simulations. In scenario 12, the peak of the epidemic was delayed by 173 days in comparison with scenario 1. 


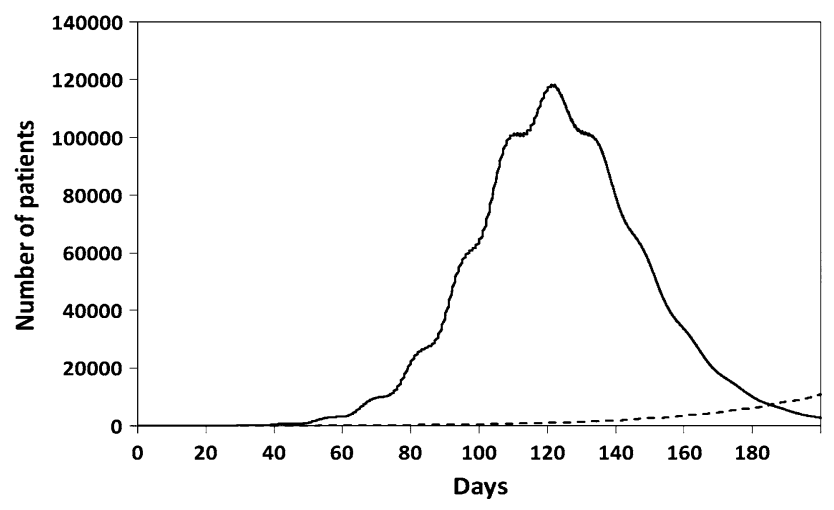

Fig. 8 Progression of the number of smallpox patients in scenarios 1 and 12. Solid and broken lines show the mean number of patients in 300 trials in scenarios 1 and 12, respectively

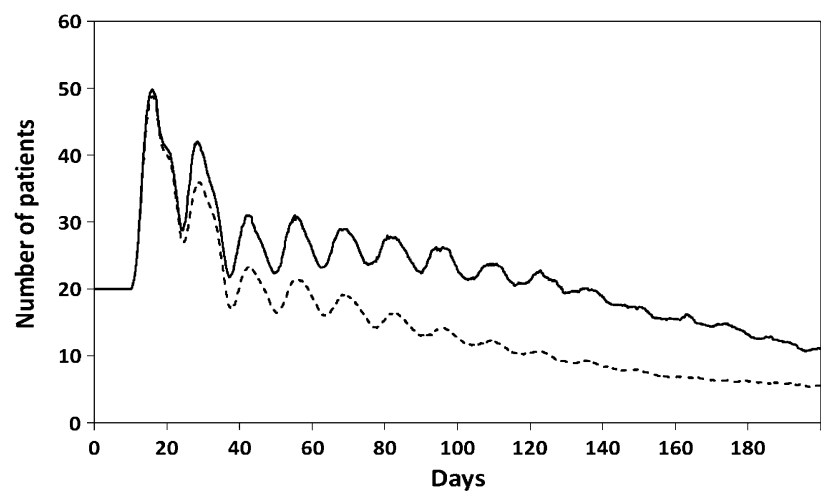

Fig. 9 Progression of the number of smallpox patients in scenarios 3 and 13. Solid and broken lines show the mean number of patients in 300 trials in scenarios 3 and 13, respectively

\section{Scenario 13}

Scenario 13 with TV accompanied by school closure was compared with scenario 3 involving only TV (Fig. 9). The mean number of total patients and deaths in scenario 13 was 124.6 and 13.0, which was lower than those in scenario 3. The success rate of elimination within 200 days for scenario 13 was assessed to be $72.0 \%$ in 300 trial simulations.

\section{Scenario 14}

Scenario 13 with MV accompanied by school closure was compared with scenario 8 involving only MV (Fig. 10). The mean number of total patients and deaths in scenario 14 was 117.1 and 25.6, which was lower than those in scenario 8. In scenario 14 , the number of patients decreased rapidly.

\section{Discussion}

This report examines simulations of a smallpox outbreak as a result of a bioterrorist attack in the center of Okayama

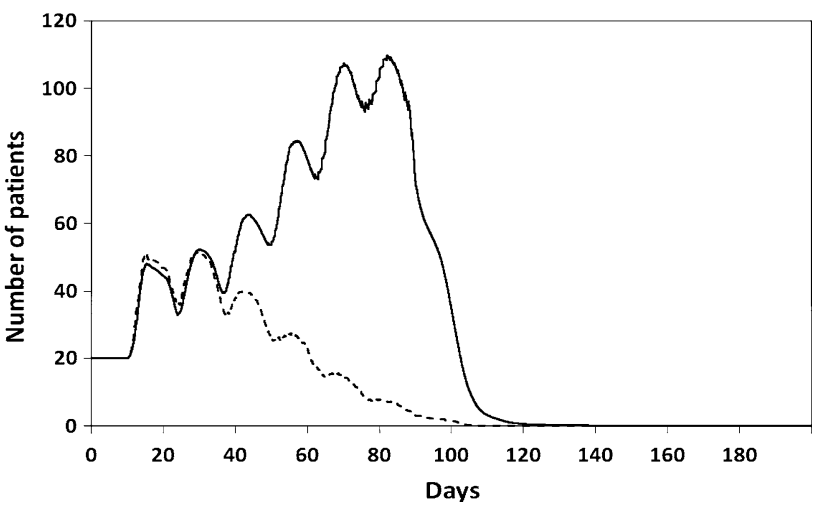

Fig. 10 Progression of the number of smallpox patients in scenarios 8 and 14. Solid and broken lines show the mean number of patients in 300 trials in scenarios 8 and 14, respectively

City using IBM, in which every individual is assigned personal information, a behavioral pattern, and interactions among social settings to simulate the situation on a realistic basis.

The center of Okayama City that has a population of about 400,000 was chosen as the target region because Okayama City, with a whole population of about 700,000, is recognized as a typical medium-sized city in Japan. The target area was divided into 37 school districts, so that each district has about 20,000 people, which is an adequate scale for a local society. It is appropriate to draw from a realistic situation because the activity of a child is limited to their own school district. Thus, the division of the targeted region into school districts fits an IBM approach.

In this article, the influence of residual immunity obtained by past vaccination allowed the model to reflect age-dependent smallpox immunity in Japan. However, assumptions on how much residual immunity could influence the severity of symptoms and the efficacy of current vaccination had to be made due to the lack of data [12].

Three types of countermeasures were considered: TV, $\mathrm{MV}$, and school closure. After the inhabitants are provided with information concerning an occurrence of bioterrorism, there is the possibility that they will be banned from leaving home [3]. It will be necessary to include this possibility among the countermeasures in further studies.

The efficacy of the scenarios in terms of the total numbers of patients and deaths, the number of vaccine doses used, and the time for eradication of the smallpox epidemic was investigated in the present study.

In the baseline scenario 1, the mean number of patients in 300 trials reached $71.4 \%$ of the entire population. As no intervention resulted in greater loss of life, it was confirmed that countermeasures would be required for rapid eradication.

Although performing TV alone (scenarios 2-4) succeeded in gradually decreasing the number of patients, it 
was difficult to eradicate the epidemic rapidly, which was because TV can trace close contacts but cannot do so for casual contacts. An advantage of TV was that fewer doses of vaccine were required for $\mathrm{TV}$ scenarios than for other scenarios. A change in the necessary tracing period from 1 to 3 days in TV was investigated. The longer tracing period was less effective, with increased numbers of patients and deaths, which confirms the necessity of prompt tracing of infected patients.

Among MV scenarios 5-10, scenario 10 with MV performed within a 2-day period at the same time in 10 school districts had the lowest loss of life, while scenario 7 with MV performed over a 1-day period in 10 school districts had difficulty eradicating the smallpox epidemic within 200 days. The difference between the 1- and 2-day administration periods (scenarios 7 and 10) of MV led to widely divergent results. The reason was the insufficient number of vaccinations administered to the entire population of each school district in only 1 day in scenario 7 due to the widespread dispersion of $\mathrm{HCW}$ to 10 school districts, while it was adequate to vaccinate most of population in the targeted school districts within 2 days in scenario 10. The number of doses of vaccine required for MV for 200 days was about 10 times as many as in TV because MV targets the entire population.

In scenario 11, performing both TV and MV simultaneously by the combination of TV scenario 4 and MV scenario 7 resulted in rapid eradication of the smallpox epidemic and a reduction in the number of patients and deaths compared with scenarios 4 or 7 , while performing $\mathrm{TV}$ for 3 days (scenario 4) or performing MV for 1 day in 10 school districts (scenario 7) could not attain eradication of the outbreak within 200 days. It was confirmed that combination of two scenarios of lower effectiveness could have higher effectiveness.

School closure alone (scenario 12) delayed the peak of the epidemic by 173 days in comparison with the baseline scenario 1 (no intervention). Even if the beginning of TV or MV was delayed, school closure could reduce loss of life. The decrease in the number of patients with TV or MV in combination with school closure was faster than that by TV or MV without school closure.

It was concluded that MV was more effective in the suppression of the number of patients and deaths and provided more rapid eradication of the smallpox epidemic than TV, and that performing both TV and MV simultaneously was much more effective than TV or MV singly and saved 100,000 or more doses in comparison with MV scenarios that succeeded in $100.0 \%$ eradication. In this article, it was assumed that countermeasures were started 5 days after diagnosis of the index patients. However, if the beginning of countermeasures was delayed, the number of patients would surely increase. School closure is performed easily and can suppress the rise in the number of patients and delay the peak of the epidemic. It is therefore advisable to carry out MV, or both TV and MV simultaneously, with school closure.

Acknowledgments This work was supported in part by a Grant-inAid from the Ministry of Health, Labor, and Welfare of Japan for "Research for emerging and re-emerging infectious diseases."

\section{References}

1. Henderson DA, Inglesby TV, Bartlett MS, Ascher MS, Eitzen E, Jahrling PB, et al. Smallpox as a biological weapon. J Am Med Assoc. 1999;281:2127-37.

2. National Institute of Infectious Diseases. Infect Dis Wkly Rep Jpn. 2001; 40:8-10 (in Japanese).

3. Ministry of Health, Labour and Welfare, Japan. The policy against smallpox (The fifth edition). Tokyo; 2004. (in Japanese).

4. Fenner F, Henderson DA, Arita I, Jezek Z, Ladnyi ID. Smallpox and its eradication. Geneve: WHO; 1988.

5. Kaplan EH, Craft DL, Wein LM. Analyzing bioterror response logistics: the case of smallpox. Math Biosci. 2003;185:33-72.

6. Center for Disease Control. CDC interim smallpox response plan and guidelines. Atlanta; 2001.

7. Nishiura H, Tang IM. Modeling for a smallpox-vaccination policy against possible bioterrorism in Japan: the impact of longlasting vaccinal immunity. J Epidemiol. 2004;14:41-50.

8. Kretzschmar M, Hof S, Wallings J, Wijngaarden J. Ring vaccination and smallpox control. Emerg Infect Dis. 2004;10:832-41.

9. Longini IM Jr, Halloran ME, Nizam A, Yang Y, Xu S, Burke DS, et al. Containing a large bioterrorist smallpox attack: a computer simulation approach. Int J Infect Dis. 2007;11:98-108.

10. Eubank S, Guclu H, Kumar VSA, Marathe MV, Srinivasan A, Toroczkal Z, et al. Modelling disease outbreaks in realistic urban social networks. Nature. 2004;429:180-4.

11. Riley S, Ferguson NM. Smallpox transmission and control: spatial dynamics in Great Britain. Proc Natl Acad Sci USA. 2006;103:12637-42.

12. Arita I. Duration of immunity after smallpox vaccination: a study on vaccination policy against smallpox bioterrorism in Japan. Jpn J Infect Dis. 2002;55:112-6.

13. Takeuchi Y, Hara M, Aoyama K. State of neutralizing antibody titer on 28 years after the abolition of vaccination against smallpox. Mod Med. 2006;52:24-7 (in Japanese).

14. Burke DS, Epstein JM, Cummings DAT, Parker JI, Cline KC, Singa RM, et al. Individual-based computational modeling of smallpox epidemic control strategies. Soc Acad Emerg Med. 2006;13:114-9.

15. Department of administration, Okayama city. The statistic of Okayama-City. Okayama; 2006 (in Japanese). 